\title{
The dissemination and implementation of national asthma guidelines in South Africa: The use of outcome mapping
}

\author{
Mash R, MBChB, MRCOG, DCH, DRCOG, PhD \\ Division of Family Medicine and Primary Care, Stellenbosch University \\ Ainslie G, MBChB, FRCP \\ Respiratory Clinic, Groote Schuur Hospital and University of Cape Town Lung Institute \\ Irusen $\boldsymbol{E}$, MBChB, $\mathrm{FCP}(\mathrm{SA})$ \\ Pulmonology Unit, Tygerberg Hospital and Department of Internal Medicine, Stellenbosch University \\ Mayers $\boldsymbol{P}$, MScMed (Psych) BA (Nursing) BCur \\ Division of Nursing and Midwifery, School of Health and Rehabilitation Sciences, University of Cape Town \\ Bheekie A, D.Pharm \\ School of Pharmacy, Discipline of Pharmacology, University of Western Cape
}

Correspondence to: Prof Bob Mash, E-mail: rm@sun.ac.za

\section{Abstract}

Asthma is an important chronic inflammatory disorder with significant morbidity and mortality in South Africa. The development of national asthma guidelines by the South African Thoracic Society and National Asthma Education Programme has been one approach to try and improve the quality of care. The effectiveness of previous guidelines has been limited by the lack of an effective approach to dissemination, implementation and evaluation. The newly revised guidelines will be completed in 2007 and this paper outlines how Outcome Mapping has been used to create a detailed and comprehensive approach to bridging the gap between the guidelines and clinical practice.

SA Fam Pract 2007;49(5): 5-8

\section{Introduction}

Asthma has been recognized as a common and important chronic disease in South Africa both in terms of its associated morbidity and mortality. . $^{1,3,4}$ At the same time numerous studies have pointed towards deficiencies in terms of clinical management, quality of care and adherence to treatment plans. 5,6 One approach to improving the quality of care has been to develop evidencebased clinical guidelines for both acute and chronic asthma in both adults and children. ${ }^{7,8,9}$ The National Asthma Education Programme (NAEP) and South African Thoracic Society (SATS) have been diligent in regularly revising these guidelines with attention to integrating new evidence.

In 2005 a meeting of respiratory physicians and asthma experts from around the country was once again held to revise the guidelines for adults. This meeting differed from previous revisions in that a number of family physicians and primary care representatives were also invited. While these participants were not expected to interpret the latest research on asthma patho-physiology and therapy they were able to reflect on the experience of receiving and using previous guidelines. These primary care participants highlighted that the effort spent revising and updating the guidelines is only worthwhile if a similar level of attention is paid to the dissemination, implementation and evaluation of the guidelines. ${ }^{10}$ Primary care providers are often bombarded with guidelines - in one study 22 general practices found they had received 855 recommended guidelines!11

Previous guidelines have mostly been disseminated through publication in scientific journals and incorporation of the key messages into continuing professional development lectures. There is good evidence that when considering ways to change professional behaviour these are amongst the most ineffective methods. ${ }^{12}$ On the other hand there is also evidence that multi-faceted interventions, educational outreach visits, reminders and interactive or participatory educational meetings may be more successful. ${ }^{12}$ There may also be a place for audit with feedback, local consensus processes, engagement of local opinion leaders and patient-mediated interventions. ${ }^{12} \mathrm{~A}$ recent review of the literature looked at the evidence for implementation of new innovations in clinical practice and found the following key principles: ${ }^{13}$

- Develop a 'good product'

- Have a systematic approach to planning

- Allow for different readiness to change amongst the target group

- Base the plan on an analysis and understanding of the target group and setting

- Be participatory in the development and implementation planning

- Use a cost-effective mix of methods - not one 'magic bullet'

- Use different strategies for dissemination and implementation

- Use different strategies for different levels - national, regional, local, individual

- Ensure continuous evaluation and revision

- Integrate implementation into existing processes, such as required audit cycles

With this in mind a team was established to develop a more comprehensive and intentional plan for the dissemination 
and implementation of the guidelines in the Western Cape. This team, which contained 2 respiratory specialists, a family physician, a nurse and a pharmacist, adopted Outcome Mapping ${ }^{14}$ as a way forward.

\section{Outcome mapping ${ }^{14}$}

Outcome mapping (www.idrc.ca) was developed by the International Development Research Centre out of the need of development projects working in complex social systems to demonstrate their contribution to a broader development goal. Outcome mapping allows projects to account for their contribution without having to prove or attribute a direct linear cause-and-effect between specific local interventions and the ultimate desired impact. This acknowledges that the ultimate goal or impact is often part of a complex social system where many factors and actors contribute. Outcomes are defined as changes in behaviour, relationships or activities amongst the project's participants rather than changes 'in state' - such as prevalence rates. Initial mapping involves systematic planning of the project as well as the associated monitoring and evaluation. The outcome mapping process is characterized as participatory, empowering and reflective with the emphasis on learning and adapting the interventions in the light of experience. Outcome mapping therefore may be useful whenever a project intends to make multiple interventions in a complex social system.

In South Africa a number of projects have utilized outcome mapping $(\mathrm{OM})$. In the Infant Feeding Research Project (www.ifrpafrica.org) OM was used to create an overall design for the project with monitoring and evaluation across 4 action research sites in 3 countries. In the Fikelela AIDS project (www.fikelela.org.za) OM was used to develop a systematic monitoring process for the interventions that were intended to change the sexual behaviour of Anglican youth in the Western Cape. At Worcester Community Health Centre OM was used to plan, monitor and evaluate organizational change that attempted to introduce practice teams of doctors and nurses. In this particular case the asthma project intends to implement new guidelines within the public and private health care systems and to contribute towards an impact on the health status of people suffering from asthma. The next sections of the article describe the steps of outcome mapping in a general way with illustrations drawn from the Asthma Guideline
Implementation Project (AGIP).

Outcome mapping has three stages as shown in Figure 1 in developing the map.

1. Intentional design

2. Outcome and performance monitoring

3. Evaluation planning

\section{Intentional design}

\section{Step 1: Vision}

Outcome Mapping begins by imagining the ultimate vision or "dream" that the project wishes to contribute towards. This is usually larger-scale and longerterm than the potential of the project alone, but enables the project to be aligned with the ultimate impact and changes that they hope to see. The vision should inspire and be a guiding light. For example:

Vision: Imagine a decrease in the severity of asthma throughout South Africa. All people who are affected by asthma are diagnosed early and well controlled on medication. Patients are able to function optimally without absence from school or work due to asthma. Hospitals notice a significant decrease in emergency care and admissions for asthma. There is a significant decrease in mortality from asthma.

Figure 1: Three stages of Outcome Mapping ${ }^{14}$

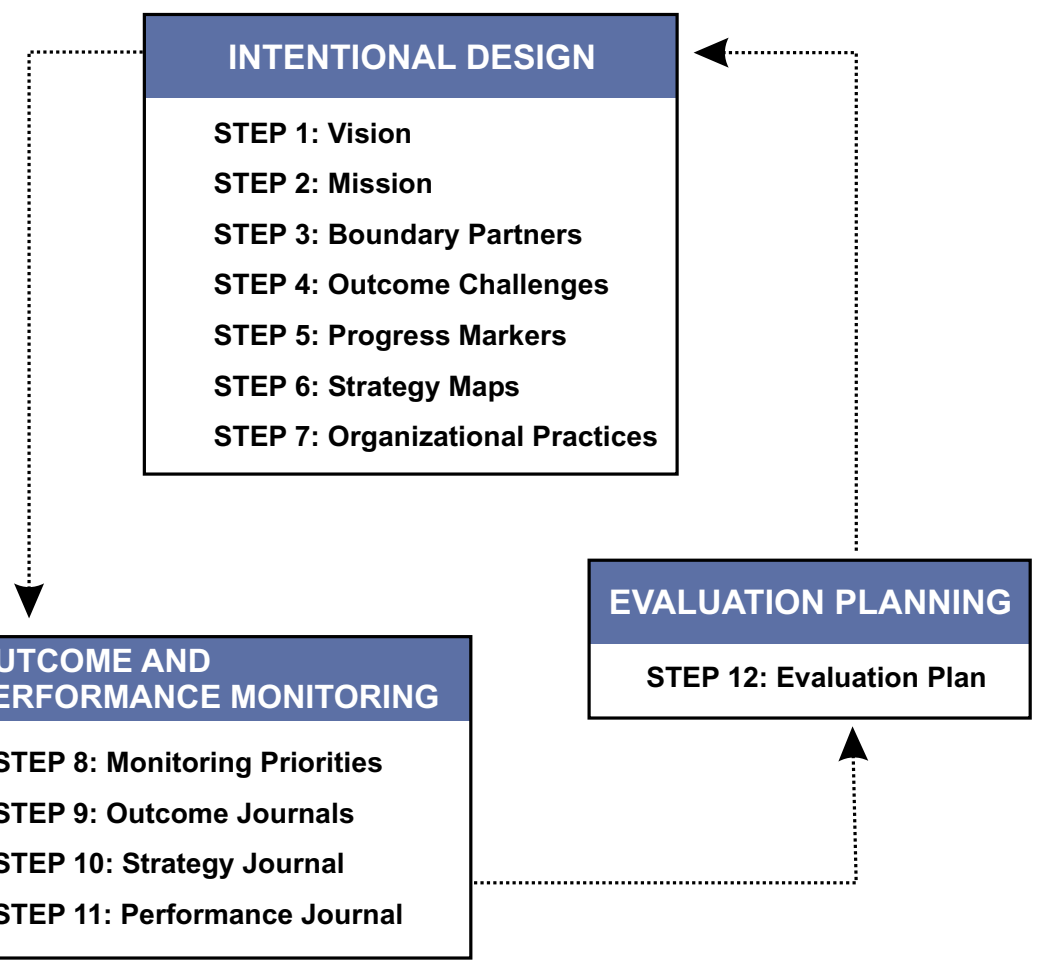

\section{Step 2: Mission}

The mission describes in more concrete terms the actual contribution that the project will make towards this broader vision. The mission describes the key areas in which the project will work under ideal or optimal performance. For example:

Mission: In support of this vision, this project aims to disseminate and implement a national asthma guideline taking cognizance of the known barriers to optimal asthma care and the successes and failures of different strategies used to influence the behaviour of health care workers and patients. All doctors, nurses and pharmacists, both state and private, especially primary care, know of NAEP, and are using the guidelines on a regular basis to both diagnose asthma early and manage it appropriately.

\section{Step 3: Boundary partners}

After defining the mission the project then identifies its boundary partners "as those individuals, groups, and organizations with whom the program interacts directly and with whom the program anticipates opportunities for influence."12 Boundary partners are not controlled by the project but influenced through the sharing of new resources, ideas, skills or opportunities. The AGIP identified seven 
Figure 2: AGIP's boundary partners

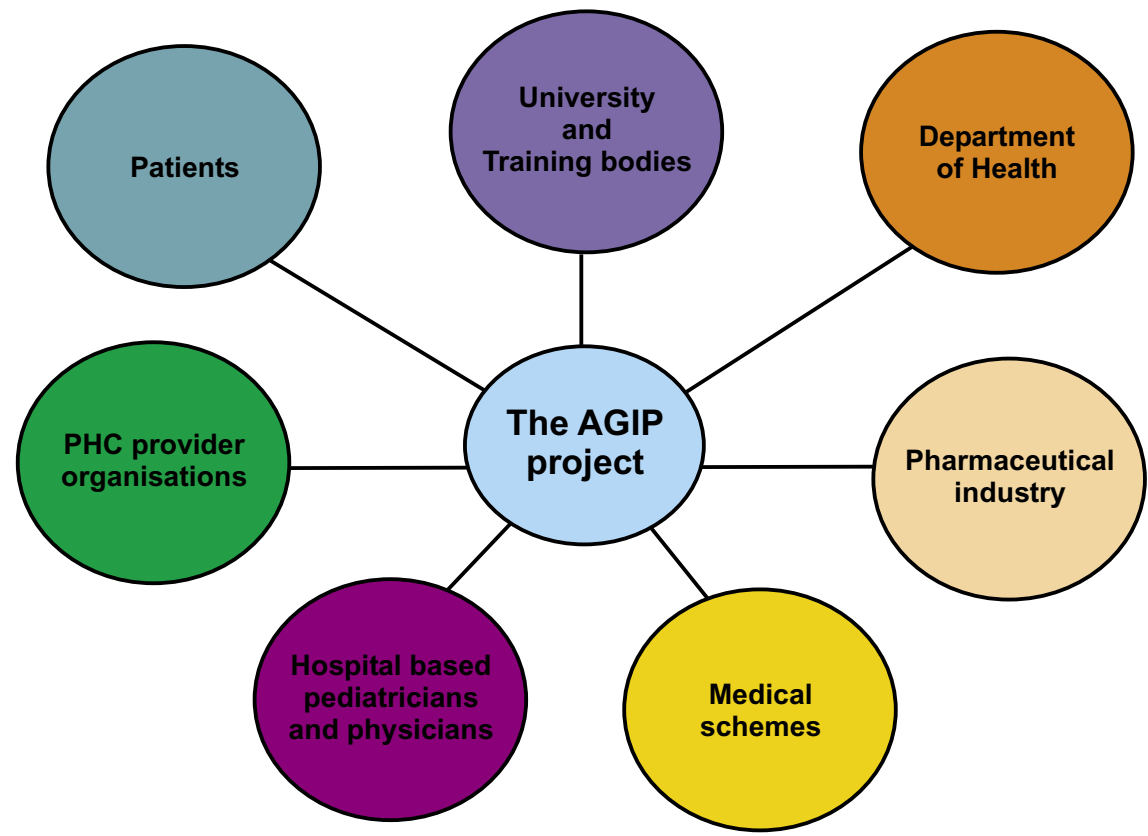

boundary partners (Figure 2) who were further broken down into specific people and organisations.

In addition to the boundary partners the project may also identify strategic partners. These are partners who are needed to make the project work, but who the project does not intend to influence in terms of its mission. The main strategic partners in this project will be organizations such as the Practical Approach to Lung Health and HIVIAIDS in South Africa (PALSA Plus) who have already created tools to guide clinical nurse practitioners in managing asthma.

\section{Step 4: Outcome challenges}

For each boundary partner the project then defines the outcome challenge that it intends to see in terms of the "changes in their behaviour, relationships, activities, or actions". ${ }^{14}$ This describes how the boundary partners will be behaving and acting if the project is very successful. For example the AGIP defined the outcome challenge for primary health care providers as:

\section{Primary health care (PHC) provi- ders}

The project intends to see that PHC workers are well informed about the diagnosis and management of asthma and have read, understood and use the guidelines on a regular basis. They recognize and diagnose asthma early, introduce anti-inflammatory medication early, assess control effectively, use objective assessment measurements, encourage use of spacers, check for inhaler technique, provide relevant information, motivate behaviour change skillfully and refer appropriately. They are aware of and use resources provided by NAEP and have access to a NAEP accredited consultant/ trainer.

\section{Step 5: Progress markers}

For each boundary partner's respective outcome challenge the project then defines a series of graduated stepping stones or progress markers under the headings of:

- "Expect to see": reactions of boundary partners to project initiatives, for example: "A key PHC worker is identified in each district who can collaborate with the project"

- "Like to see": change in activities of boundary partners, for example "PHC workers in each district are using one of the project's tools in their facilities"

- "Love to see": deeper changes in values, goals or beliefs of boundary partners, for example: "PHC workers are using the guideline messages in patient care on a regular basis"

\section{Step 6: Strategies}

In order to achieve each of these outcome challenges the project then considers what strategies or activities are necessary. Strategies may be aimed at specific individuals, groups or the environment within which they operate. The strategies initially considered for the primary health care providers were:

\section{Primary health care providers}

1. The project will publish the guidelines in a manual format that is short, practical, easy-to-follow, and targeted at specific professionals in specific contexts. The manual will be piloted with the key target audiences and revised based on their feedback. Where possible the manual will be integrated with other local initiatives of this nature.

2. The project will use reminders of the key messages, for example in the form of a desktop tool or posters.

3. The NAEP distance learning diploma (SA Diploma in Asthma Care) will be promoted to and subsidized for key providers and opinion leaders in each sub-district.

4. The project will create a framework for and resources to support interactive training workshops aimed at health care workers. Co-trainers from the primary care provider organizations will be identified and trained in how to facilitate the workshop in their setting. Resources would include patient education materials (print or video), guideline manual(s), reminders, video material on motivational interviewing (adherence) skills, audit tools, and peak flow meters.

5. The project will create generic material to support simple quality assessment and improvement activities (audit) that support the key guideline messages and quality criteria.

6. The project will publish the guidelines in the format of journal articles and negotiate for an edition of the CME Journal on asthma care in 2008.

\section{Step 7: Organisational practices}

This step involves listing the key organizational practices that are expected of the project team itself in order to function optimally. For example "experimenting to remain innovative" or "obtaining the support of your next highest power". ${ }^{14}$ 
